\title{
Cuerpos extraños uretrovesicales: Nuestra experiencia
}

\author{
M. Bosquet Sanz, V. Gimeno Argente, J.L. Palmero Martí, M.A. Bonillo García, \\ S. Arlandis Guzmán, J.F. Jiménez Cruz \\ Servicio de Urología. Hospital Universitario La Fe. Valencia.
}

Actas Urol Esp 2005; 29 (6): 572-577

\section{RESUMEN}

CUERPOS EXTRAÑOS URETROVESICALES: NUESTRA EXPERIENCIA

Realizamos una revisión retrospectiva de doce pacientes con cuerpo extraño uretral y vesical que hemos atendido en nuestro servicio desde 1976 hasta septiembre del 2004.

Analizamos el tipo de cuerpo extraño, características del paciente, incluyendo su perfil psicológico, clínica con la que se presenta, su diagnóstico y tratamiento. Realizamos una revisión de la literatura al respecto.

Palabras clave: Cuerpos extraños uretro-vesicales.

\section{ABSTRACT}

FOREIGN BODIES IN THE URETHRA AND BLADDER: OUR EXPERIENCE

We report the urethra and bladder foreign bodies seen in our department from 1976 to September 2004.

We review the kind of the foreign bodies, the psychological profile of the patient, the clinical findings, diagnosis and treatment. We also review the literature about this matter.

Key words: Foreign bodies in urethra and bladder.

$\mathrm{E}^{\mathrm{l}}$ hallazgo de cuerpos extraños en uretra y ejiga es una circunstancia relativamente poco frecuente. La mayoría de cuerpos extraños auto introducidos se producen en un ambiente erótico-sexual por lo que muchos pacientes intentan ocultarlo o incluso intentan retrasar la ayuda médica hasta que la clínica no sea lo suficientemente intensa. Es por ello que debemos estar alerta ante síntomas que no podemos encuadrar en otra patología y sobretodo ante pacientes deficientes mentales, en los que esta entidad se asocia con bastante frecuencia. La forma de presentación puede ser muy variada en función del tamaño del objeto y de la localización del mismo, pudiendo debutar con una clínica aguda, sobre todo los localizados en uretra, o una clínica subaguda o crónica, los localizados en vejiga.

Con motivo de un caso reciente de auto introducción de un cuerpo extraño uretral hemos revisado retrospectivamente todos los casos de cuerpo extraño uretral y vesical en adultos que hemos atendido en nuestro servicio desde el año 1976 hasta septiembre de 2004, con revisión de la literatura publicada al respecto.

\section{MATERIAL Y MÉTODOS}

Son 12 pacientes cuyas edades van desde los 15 años hasta los 75, con una edad media de 39. No incluimos niños, puesto que son atendidos en la Unidad de Urología Pediátrica.

El último caso de la serie es un varón de 38 años de edad, que como antecedente destaca que fue intervenido de hipospadias hace unos veinte años, que acudió a urgencias refiriendo la introducción de un pajita en la uretra. Según el paciente, la finalidad fue la dilatación del meato uretral, pues se dilataba el meato con sondas uretrales pero cuando no disponía de ellas utilizaba estos artículos para dilatarse sin haber tenido ningún problema hasta la fecha. A la exploración física se observó un pene edematizado, sobretodo a nivel del tercio medio en su cara ventral donde se podía palpar el extremo distal del 
cuerpo extraño (Fig. 1). No se palpaba globo vesical. Se procedió a la extracción urgente del cuerpo extraño (una pajita), localizado en uretra peneana e impactado en uretra bulbar, mediante pinzas bajo visión con uretrocistoscopio (Fig. 2). No conocemos la evolución posterior por no acudir a consultas externas en la fecha prevista.

De los 12 pacientes, tan sólo uno es mujer y el resto son todo varones.

Los objetos alojados son de muy diversa índole como se detalla en la Tabla 1.

Cinco de ellos fueron auto introducidos con fines masturbatorios (todos con un perfil psicológico normal a excepción de un paciente que presentaba demencia), en tres la finalidad por la que

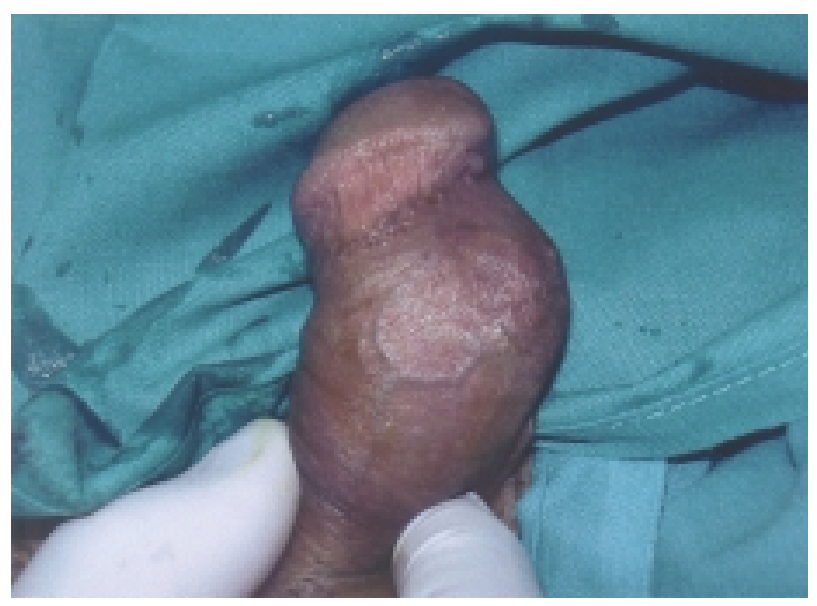

FIGURA 1. Imagen en la que se puede apreciar una zona edematosa a nivel de la base del pene y en la que se podia palpar el extremo del cuerpo extraño.

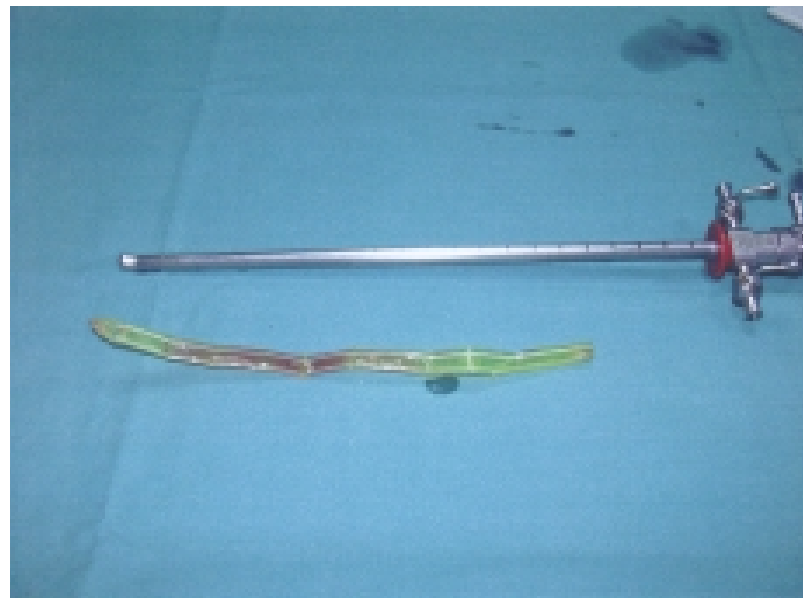

FIGURA 2. Imagen en la que podemos observar el cuerpo extraño (una pajita) ya extraído, comparándolo con el cistoscopio.
Tabla 1

Tipo de cuerpo extraño y localización

\begin{tabular}{ccccc}
\hline Paciente & Sexo & Edad & Tipo de cuerpo extraño & Localización \\
\hline 1 & Varón & 15 & Cable eléctrico & Uretra-vejiga \\
2 & Varón & 65 & Tubo de plástico & Vejiga \\
3 & Varón & 15 & Alambre & Vejiga \\
4 & Mujer & 17 & Guijarro & Vejiga \\
5 & Varón & 64 & Tubo de plástico & Vejiga \\
6 & Varón & 75 & Dilatador uretral & Vejiga \\
7 & Varón & 41 & Pinzas, horquilla, chincheta & Uretra \\
8 & Varón & 48 & Clip sujetapapeles & Uretra \\
9 & Varón & 38 & Funda de plástico de boligrafo & Uretra \\
10 & Varón & 22 & Fragmento de pubis calcificado & Vejiga \\
11 & Varón & 37 & Hoja de pino & Uretra \\
12 & Varón & 38 & Pajita & Uretra \\
\hline
\end{tabular}

fue introducido el cuerpo extraño no fue aclarada porque eran oligofrénicos, la mujer padecía depresión y se introdujo un guijarro sin saber cuál fue el motivo que la impulsó, y en dos casos, aunque los motivos que justificaron los pacientes en la anamnesis eran otros, sospechamos que el verdadero motivo fue el masturbatorio (Tabla 2).

Sólo en un caso el cuerpo extraño no fue auto introducido:

Era un varón de 22 años de edad que, tras sufrir dos años antes un politraumatismo por accidente de tráfico en el que presentó una rotura vesical extraperitoneal que precisó sondaje

\section{Tabla 2}

Objetivos y perfil psicológico

\begin{tabular}{ccc}
\hline Paciente & Objetivos & Perfil psicológico \\
\hline 1 & Masturbatorio & Normal \\
2 & Masturbatorio & Normal \\
3 & Desconocido & Oligofrénico \\
4 & Desconocido & Depresión \\
5 & Masturbatorio & Normal \\
6 & Masturbatorio & Demencia \\
7 & Desconocido & Oligofrénico \\
8 & Desconocido & Oligofrénico \\
9 & Extracción cálculo uretra & Normal \\
10 & - & Normal \\
11 & Masturbatorio & Normal \\
12 & Dilatación meato uretra & Normal \\
\hline
\end{tabular}


vesical, acudió con clínica miccional irritativa con infecciones urinarias de repetición, alguna de ellas complicada con prostatitis aguda, y urinocultivos y cultivos de semen positivos a E. coli y Enterococcus faecalis resistente a múltiples antibióticos. Tras varios meses de tratamiento antibiótico infructuoso y ante la persistencia de la clínica se llevó a cabo una cistoscopia en la que se evidenció un fragmento de pubis calcificado incrustado en la vejiga que precisó la realización de una cistolitotomía para su tratamiento.

El motivo de consulta más frecuente fue el escozor al orinar, presente en seis de los doce casos. De estos seis pacientes cuatro asociaban hematuria, que era el único síntoma en un paciente. La infección urinaria fue demostrada en dos casos. Un paciente presentó clínica obstructiva y dos consultaron por dolor (Tabla 3).

Tabla 3

Clínica y tratamiento

\begin{tabular}{ccc}
\hline Paciente & Clínica & Tratamiento \\
\hline 1 & Dolor y tenesmo & Cistotomía \\
2 & Disuria y hematina & Cistotomía \\
3 & Hematuria & Cistotomía \\
4 & Cistitis - ITU & Endoscópico \\
5 & Disuria & Endoscópico \\
6 & Disuria y hematuria & Endoscópico \\
7 & Disuria & Endoscópico \\
8 & Disuria y hematuria & Endoscópico \\
9 & Uretrorragia y dificultad para orinar & Meatotomía \\
10 & ITUs de repetición & Cistolitotomia \\
11 & Disuria y hematuria & Endoscópico \\
12 & Dolor & Endoscópico \\
\hline
\end{tabular}

Respecto a la localización, en seis casos el cuerpo extraño se encontraba en la vejiga, en cinco casos se encontraba en uretra y en uno, dado que el cuerpo extraño era un cable eléctrico de varios centímetros de longitud, abarcaba vejiga y uretra.

El perfil psicológico estaba alterado en cinco pacientes:tres eran oligofrénicos, uno presentaba demencia y una mujer, depresión. En el resto no se observaron alteraciones mentales destacables.

Por último, respecto al tratamiento, en siete fue endoscópico, que se llevó a cabo en cuatro de los cinco casos de cuerpo extraño uretral y en tres de los de cuerpo extraño vesical, y tan sólo en cinco casos se tuvo que recurrir a la cirugía abierta, practicándose una meatotomía en un paciente que se auto introdujo una funda de plástico de bolígrafo en uretra y en el resto se realizó una cistotomía para extraer el cuerpo extraño, incluyendo el caso del cable eléctrico que antes comentábamos.

\section{DISCUSIÓN}

Como hemos comentado no existen muchos casos para un tiempo de seguimiento evolutivo tan largo (28 años), lo que hace pensar que los cuerpos extraños uretro-vesicales no representan una patología frecuente en la práctica clínica diaria; no obstante las referencias bibliográficas en la literatura son abundantes, la mayoría publicando casos clínicos aislados o complicaciones de los mismos. Nosotros publicamos en 1991 una serie con ocho casos ${ }^{1}$, desde entonces, en catorce años, añadimos cuatro casos más.

Cuando nos preguntamos el motivo por el que aparece un cuerpo extraño en la vejiga o en la uretra casi siempre pensamos que ha sido la auto introducción voluntaria con fines masturbatorios, sin embargo no es el único motivo aunque sí uno de los más frecuentes.

Una vez revisada la literatura, la variedad de cuerpos extraños con que nos podemos encontrar es mayúscula y hace necesaria una clasificación para así tratar de agrupar aquéllos que tienen un mecanismo similar. Según su origen, los cuerpos extraños se podrían clasificar en dos grandes grupos:de una parte los introducidos desde el exterior y de otra los que se albergan en la vejiga o en la uretra migrados desde otros lugares ${ }^{2}$.

Los cuerpos extraños introducidos desde el exterior quedarian clasificados a su vez en tres grupos:

- Los cuerpos extraños auto introducidos. Es sin duda el grupo más frecuente y en el que podemos apreciar la gran variedad y naturaleza de los objetos que nos podemos encontrar en el tracto urinario inferior. Buena muestra de ello es la serie que acabamos de presentar.

- Los que se alojan en la uretra o la vejiga de forma yatrógena. Incluimos en este grupo todos aquellos objetos que quedan alojados en el tracto urinario inferior después de una manipulación ya sea endoscópica o abierta. Así pues podemos 
encontrar dilatadores uretrales, catéteres ureterales doble $\mathrm{J}$, puntos de sutura y agrafes, fragmentos de material endoscópico, etc. Más raro, aunque descrito, es encontrar gasas quirúrgicas ${ }^{3}$.

- Los que encontramos después de traumatismos. Es un grupo poco frecuente y en el que los cuerpos extraños quedan alojados en el tracto urinario inferior después de un mecanismo traumático o accidental:fragmentos óseos, metralla, proyectiles como perdigones o balas ${ }^{4,5}$, etc.

De otra parte tenemos los cuerpos extraños migrados desde otros lugares, que a su vez los clasificamos en función de su origen ${ }^{2}$ :

- De origen urológico:partes de prótesis antiincontinencia, componentes de prótesis de pene, agrafes, etc.

- De origen ginecológico:DIUs ${ }^{6}$, etc.

- De origen digestivo:alimentos no digeridos como espinas de pescado, materiales quirúrgicos como agrafes o mallas, etc.

- De origen vascular:prótesis vasculares, etc.

Una vez hecha una clasificación de los cuerpos extraños en función de su origen, otro aspecto importante a tener en cuenta serían las características del paciente. Si excluimos aquellos que han sido sometidos a una cirugia previa, ya sea urológica o no, o que han sufrido un traumatismo, tan sólo nos quedan los pacientes en que los cuerpos extraños han sido auto introducidos.

Como comentábamos, importante por su alta frecuencia es la asociación con alteraciones mentales o psiquiátricas, como ocurre en nuestra serie, con fines principalmente erótico-sexuales o auto lesivos. Cabe tener en cuenta en estos pacientes que son maniobras reiterativas y en las que en muchas ocasiones nos podemos encontrar varios cuerpos extraños, alguno de ellos de mucho tiempo de evolución ${ }^{7}$, ya que, si la sintomatología es poco intensa, los pacientes tienden a buscar ayuda médica de forma $\operatorname{tardía}^{8}$ y eso dificulta mucho el diagnóstico.

En pacientes que no presentan tales alteraciones es frecuente que el cuerpo extraño quede alojado en la uretra en el varón o en la vejiga en la mujer después de un accidente en el contexto de maniobras masturbatorias o juegos sexuales entre las parejas.
Pacientes de riesgo consideraríamos también a los niños que movidos por la curiosidad y con fines auto exploratorios son susceptibles de auto introducirse estos cuerpos extraños. Otros pacientes de riesgo serian aquellos que, bajo el efecto de las drogas y con fines sexuales, introducen cuerpos extraños, a sí mismos o a su pareja, o personas encarceladas en el contexto de maniobras evasivas ${ }^{2}$.

Respecto a la clínica, la forma de presentación se clasifica en función de la localización del cuerpo extraño:los alojados en la uretra producen una clínica aguda y subaguda o crónica los cuerpos intravesicales $^{1}$. Teniendo en cuenta las alteraciones mentales subyacentes en muchos pacientes y que muchos cuerpos extraños se han introducido en un ambiente erótico-sexual, es fácil entender que se retrase la atención médica hasta que se presente la clínica o, aun relatando los síntomas se intente esconder la introducción de un cuerpo extraño o el fin por el cual fue introducido. Es por ello que en muchas ocasiones hay que sospechar su presencia cuando los síntomas no son del todo claros.

Junto a estos pacientes con alteraciones mentales o que intentan esconder la introducción del cuerpo extraño, hay otros en los que el diagnóstico no es fácil porque alojan cuerpos extraños migrados desde otros lugares o que han quedado alojados de forma yatrógena. Es por ello que una de las formas más frecuentes de presentarse un cuerpo extraño es como hallazgo casual.

Otra forma importante de presentación es como litiasis o calcificación del cuerpo extraño. Aunque es infrecuente que un cuerpo extraño actúe de nido de formación de un cálculo por la severidad en los síntomas urinarios que suele producir inmediatamente a su introducción, sí que suele ocurrir en aquellos en que el cuerpo extraño queda alojado en el tracto urinario inferior sin producir clínica y por un largo periodo de tiempo, con calcificación tardía, provocando síntomas que van a depender del tamaño y de su localización. Cabe tener en cuenta que los cálculos uretrales primarios son extremadamente raros por lo que deberíamos sospechar la existencia subyacente de un cuerpo extraño ante su hallazgo ${ }^{9}$.

Los cuerpos extraños que no se presentan como hallazgo casual y sí que producen síntomas lo pueden hacer de una forma muy variada: 
- Como un síndrome miccional obstructivo o como cuadro de retención aguda de orina, sobretodo los alojados en la uretra.

- Síndrome miccional más o menos intenso, con urgencia miccional, disconfort pélvico o dolor hipogástrico y tenesmo vesical.

- Infecciones urinarias de repetición, o prostatitis agudas recidivantes en el varón, provocadas en ciertas ocasiones por más de un patógeno y/o patógenos multirresistentes. Tal es el caso del paciente con traumatismo pélvico en el que quedó incrustado un fragmento óseo intravesical posteriormente calcificado y en el que se aisló un Enterococcus faecalis en sucesivos urinocultivos. También se han publicado infecciones por Proteus mirabillis o Pseudomona aeruginosa ${ }^{2}$ asociadas a cuerpos extraños. En casos más evolucionados incluso se podría presentar en forma de absceso que engloba el cuerpo extraño.

- Hematuria o uretrorragia.

- Otros:insuficiencia renal aguda obstructiva, expulsión espontánea de cuerpos extraños ${ }^{5}$, signos de fístula entero-vesical o vésico-vaginal ${ }^{2}$.

Respecto al diagnóstico, en aquellos pacientes que acuden por voluntad propia suele ser suficiente con la anamnesis y la exploración física ya que suelen admitir la introducción del cuerpo extraño. No obstante, y como comentábamos antes, hay que tener en cuenta las circunstancias especiales de algunos pacientes, lo que hace que la historia clínica pueda llegar a ser dificultosa, llena de contradicciones e incluso a veces con tendencia al engaño ${ }^{7}$. Cabe realizar una exploración física minuciosa tanto urológica como genital a fin de descartar la existencia de lesiones asociadas y en algunos casos puede ser de ayuda la realización de una radiografía simple de abdomen que incluya vejiga y uretra.

En los casos en que a pesar de la anamnesis, la exploración física y la radiografía simple el diagnóstico continúe incierto habrá que recurrir a otras pruebas complementarias como la ecografía, la CUMS, la UIV (en casos que se sospeche uropatía obstructiva) o la uretrocistoscopia que es la prueba que nos dará el diagnóstico de confirmación, además de permitir la extracción del cuerpo extraño. Puede ocurrir también que el diagnóstico sea posterior al tratamiento, como sucede en litiasis vesicales o uretrales en que se evidencia, posteriormente a su extracción, que el cálculo se ha formado a partir de un cuerpo extraño que ha servido de núcleo para su formación $^{3,6,9}$.

Un aspecto importante que se plantea en el diagnóstico es la evaluación psiquiátrica del paciente ante el hallazgo de un cuerpo extraño auto introducido. Probablemente la causa más frecuente sea la auto introducción voluntaria con fines erótico-sexuales, ya sea mediante masturbación $u$ otras variantes de estimulación sexual $^{10}$. Según Kenney estas maniobras serían llevadas a cabo por individuos con un perfil psicológico completamente normal inducidos por el placer que produce la estimulación de la mucosa uretral, siendo ésta el principio de la introducción completa del cuerpo extraño. No obstante esta acción de prevalencia desconocida podría estar indicando una predisposición psicológica particular en estos individuos, en los que se combinarian rasgos sadomasoquistas y fetichistas debido, desde el punto de vista de la teoría psicoanalítica, a una regresión a la etapa uretral de la sexualidad producida por ciertos eventos traumáticos o conductas sexuales inadecuadas que modificarían el desarrollo psicosexual normal ${ }^{11}$. Se ha descrito en la literatura la introducción de cuerpos extraños en el contexto de maniobras auto lesivas como tentativas de suicidio, por lo que deberíamos estar atentos a la existencia en el paciente de maniobras impulsivas, conductas regresivas o estados depresivos en las que deberíamos demandar ayuda de un psiquiatra, pues en la mayoría de estos casos estaría indicada la hospitalización en un servicio de psiquiatría ${ }^{10}$.

El tratamiento dependerá de:la localización del cuerpo extraño, su tamaño, forma y movilidad, el sexo y edad del paciente, y la existencia de lesiones asociadas. Una vez analizadas estas características, y siempre que sea posible, la vía más recomendada para la extracción del cuerpo extraño es la endoscópica, teniendo que recurrir a la vía abierta en contadas ocasiones.

La extracción endoscópica transuretral es quizás el método más fisiológico, con menos complicaciones que el abordaje abierto, puede realizarse sin necesidad de anestesia y tiene una recuperación postoperatoria más rápida. En las mujeres el tratamiento endoscópico plantea menos problemas que 
en el varón, pues la anatomía de la uretra masculina o la existencia de una impronta prostática importante dificultan la maniobra y precisa, en muchas ocasiones, al menos de una sedación ${ }^{2}$.

$\mathrm{Si}$ el cuerpo extraño es muy grande, impactado en la uretra, de bordes irregulares o con aristas cortantes que podrían poner en peligro la integridad de la mucosa uretral o esfinteriana y suponer el desarrollo ulterior de estenosis o incontinencia urinaria, o que presenta lesiones asociadas o zonas abscesificadas, sería preferible desestimar la extracción transuretral y llevar a cabo otras técnicas como las percutáneas a partir de la vejiga o las abiertas.

$\mathrm{El}$ abordaje percutáneo se efectúa a través de una punción vesical suprapúbica en la que, tras dilatación progresiva, se introduce un cistoscopio mediante el que efectuamos la extracción, minimizando así el daño a la mucosa uretral ${ }^{12}$. También podríamos utilizar técnicas combinadas en las que introduciriamos a partir de una cistostomía percutánea un puerto de laparoscopia que actuaría de canal de trabajo bajo visión endoscópica transuretral directa con un uretrocistoscopio ${ }^{13,14}$

La cirugía abierta, ya sea mediante laparotomía y cistotomía o mediante uretrotomía o meatotomía, la reservaríamos como último recurso cuando por las características del cuerpo extraño o la severidad de las lesiones asociadas, tales como la presencia de fístulas o abscesos, obligaría a desestimar el abordaje endoscópico transuretral o las técnicas percutáneas.

Abandonadas han quedado técnicas de antaño que consistían en instilar benceno, queroseno, $\mathrm{u}$ otras sustancias químicas similares con la intención de disolver el cuerpo extraño y así facilitar su expulsión ${ }^{10}$.

\section{CONCLUSIONES}

La presencia de cuerpos extraños en el tracto urinario inferior es una entidad infrecuente, asociada con cierta frecuencia a alteraciones mentales, y cuya causa más importante es la auto introducción voluntaria con fines erótico-sexuales. Otras causas serían la migración de cuerpos extraños, los traumatismos y la yatrogenia.

La presentación clínica puede ser muy variada, desde cuadros agudos en forma de retención aguda de orina hasta cuadros de larga evolución en forma de síndrome miccional irritativo, siendo la forma más importante de presentación el hallazgo casual.

El diagnóstico resulta en ocasiones muy difícil, tanto por las características del paciente como por la causa que motivó la introducción del cuerpo extraño, con una anamnesis dificultosa y a veces con tendencia al engaño. Sería interesante hacer una valoración psiquiátrica a aquellos pacientes con conductas de riesgo.

La prueba diagnóstica de confirmación es la uretrocistoscopia, que permitiría llevar a cabo además la extracción del cuerpo extraño, considerando este tratamiento de elección por ser el menos cruento, con menos complicaciones, utilizar la vía más fisiológica y tener una recuperación postoperatoria más rápida. Otras técnicas a considerar serían las percutáneas, las combinadas con laparoscopia y la cirugía abierta.

\section{REFERENCIAS}

1. Osca JM, Broseta E, Server G, Ruiz JL, Gallego J, Jiménez Cruz JF Unusual Foreign Bodies in the Urethra and Bladder. $\mathrm{Br} \mathrm{J}$ Urol 1991;68:510-512.

2. Pascual D, García de Jalón A, Mallén E, Sancho C, Gonzalo A, Rioja LA. Cuerpos extraños intravesicales. Revisión de la literatura. Actas Urol Esp 2003;27:265-273.

3. Recasens JR, Flores JJ, Samsó JM, Ribes Y, Massó A. Litiasis vésicouretral secundaria a cuerpo extraño. Actas Urol Esp 2002;26:136-138.

4. Gulanikar A, Pandey P, Terrell F. A bullet in the bladder. Br J Urol 1998;82:304.

5. Sankari BR, Parra RO. Spontaneous voiding of a bullet after gunshot wound to the bladder: case report. J Trauma 1993;35:813-814.

6. Hermida JA, Del Corral T, Cerdeiras G, Agüero JL, Machado V. Litiasis vesical formada a partir de un DIU (Dispositivo Intrauterino). Un caso raro. Arch Esp de Urol 1997;50:808-809.

7. Briones G, Jiménez M, Sáenz J, Pozo B, Sanz JL, Cruz N. Cuerpos extraños uretro-vesicales. Actas Urol Esp 2001;25:456-457.

8. García V, Vareal M, Fernández L. Cuerpos extraños de uretra. A propósito de dos casos. Arch Esp de Urol 1999;52:74-76.

9. Noble JG, Chapple CR. Formation of a Urethral Calculus around an Unusual Foreign Body. Br J Urol 1993;72:248-249.

10. Van Ophoven A, De Kernion JB. Clinical management of foreign bodies of the genitourinary tract. J Urol 2000;164:274-287.

11. Kenney RD. Adolescent males who insert genitourinary foreign bodies: is psychiatric referral required? Urology 1988;32:127-129.

12. Johnin K, Kushima M, Koizumi Sh, Okada Y. Percutaneous transvesical retrieval of foreign bodies penetrating the urethra. J Urol 1999; 161:915-916

13. Hutton KA, Huddart SN. Percutaneous retrieval of an intravesical foreign body using direct transurethral visualization: a technique applicable to small children. Br J Urol 1999;83:337-338.

14. Bukowski TP, Williams CR. Percutaneous intravesical retrieval of a misplaced urethral stent: new laparoscopic instrumentation. J Urol 1997; 157:2257.

Dr. M. Bosquet Sanz

Servicio de Urología. Hospital Universitario La Fe

Avda. Campanar 21 - 46009 Valencia

(Trabajo recibido el 30 marzo 2005) 